\title{
The retrograde orbit of the HAT-P-6b exoplanet ${ }^{\star, \star \star}$
}

\author{
G. Hébrard ${ }^{1,2}$, D. Ehrenreich ${ }^{3}$, F. Bouchy ${ }^{1,2}$, X. Delfosse ${ }^{3}$, C. Moutou ${ }^{4}$, L. Arnold ${ }^{2}$, I. Boisse ${ }^{5,1}$, X. Bonfils ${ }^{3}$, \\ R. F. Díaz ${ }^{1,2}$, A. Eggenberger ${ }^{3}$, T. Forveille ${ }^{3}$, A.-M. Lagrange ${ }^{3}$, C. Lovis ${ }^{6}$, F. Pepe ${ }^{6}$, C. Perrier ${ }^{3}$, D. Queloz ${ }^{6}$, \\ A. Santerne ${ }^{4}$, N. C. Santos ${ }^{5,7}$, D. Ségransan 6 , S. Udry ${ }^{6}$, and A. Vidal-Madjar ${ }^{1}$ \\ 1 Institut d'Astrophysique de Paris, UMR7095 CNRS, Université Pierre \& Marie Curie, 98bis boulevard Arago, 75014 Paris, France \\ e-mail: hebrard@iap.fr \\ 2 Observatoire de Haute-Provence, CNRS/OAMP, 04870 Saint-Michel-1'Observatoire, France \\ 3 UJF-Grenoble 1/CNRS-INSU, Institut de Planétologie et d'Astrophysique de Grenoble (IPAG), UMR 5274, 38041 Grenoble, \\ France \\ ${ }^{4}$ Laboratoire d'Astrophysique de Marseille, Univ. de Provence, CNRS (UMR6110), 38 rue F. Joliot Curie, 13388 Marseille Cedex \\ 13, France \\ 5 Centro de Astrofísica, Universidade do Porto, Rua das Estrelas, 4150-762 Porto, Portugal \\ 6 Observatoire de Genève, Université de Genève, 51 Chemin des Maillettes, 1290 Sauverny, Switzerland \\ 7 Departamento de Física e Astronomia, Faculdade de Ciências, Universidade do Porto, Portugal \\ Received 15 December 2010 / Accepted 26 January 2011
}

\section{ABSTRACT}

\begin{abstract}
We observed the transit of the HAT-P-6b exoplanet across its host star with the SOPHIE spectrograph (OHP, France). The resulting stellar radial velocities display the Rossiter-McLaughlin anomaly and reveal a retrograde orbit: the planetary orbital spin and the stellar rotational spin point in approximately opposite directions. A fit to the anomaly measures a sky-projected angle $\lambda=166^{\circ} \pm 10^{\circ}$ between these two spin axes. All seven known retrograde planets are hot Jupiters with masses $M_{\mathrm{p}}<3 M_{\text {Jup }}$. About two thirds of the planets in this mass range, however, are prograde and aligned $\left(\lambda \simeq 0^{\circ}\right)$. In contrast, most of the more massive planets $\left(M_{\mathrm{p}}>4 M_{\text {Jup }}\right)$ are prograde but misaligned. Different mechanisms may therefore be responsible for planetary obliquities above and below $\sim 3.5 M_{\mathrm{Jup}}$.
\end{abstract}

Key words. planets and satellites: dynamical evolution and stability - techniques: radial velocities - planetary systems stars: individual: HAT-P-6

\section{Introduction}

Spectroscopic observations during the transit of an exoplanet across its host star can measure the sky-projected angle between the spins of the planetary orbit and the stellar rotation (the obliquity) through the Rossiter-McLaughlin (RM) effect (Holt 1893; Rossiter 1924; McLaughlin 1924). The occultation of a rotating star by a planet distorts the apparent stellar line shape by removing the profile part emitted by the hidden portion of the star. This induces anomalous stellar radial velocity variations during the transit, which constrain the sky-projected obliquity $(\lambda)$, thus indicating whether the orbit is prograde, retrograde, or polar.

Queloz et al. (2000) reported the first detection of the RM anomaly for an extrasolar planet, HD 209458b. That planet shows an aligned, prograde orbit, as did all of the first seven planets for which the RM effect was measured (as reviewed in Hébrard et al. 2008). These early results were interpreted as validating theories of planetary formation and evolution where a single giant planet migrates in a proto-planetary disk perpendicular to the stellar spin axis (e.g. Lin et al. 1996). That migration is expected to conserve the initial alignment between the angular momentums of the disk and of the planetary orbits.

* Based on observations collected with the SOPHIE spectrograph on the 1.93-m telescope at Observatoire de Haute-Provence (CNRS), France, by the SOPHIE Consortium (program 10A.PNP.CONS).

$\star \star$ SOPHIE radial velocities are only available in electronic form at the CDS via anonymous ftp to cdsarc.u-strasbg.fr (130.79.128.5) or via

http://cdsarc.u-strasbg.fr/viz-bin/qcat?J/A+A/527/L11
Hébrard et al. (2008), however, found a first case of spinorbit misalignment for the XO-3b planet, confirmed by Winn et al. (2009a). Thereafter, Moutou et al. (2009) reported a second case, HD 80606 (see also Pont et al. 2009; Winn et al. 2009b; Hébrard et al. 2010). A dozen misaligned systems have now been identified, including some with retrograde or nearly polar orbits (e.g. Winn et al. 2009c; Narita et al. 2010; Triaud et al. 2010; Simpson et al. 2010). These unexpected results favor alternative scenarios where close-in massive planets have been brought in by planet-planet (or planet-star) scattering, Kozai migration, and/or tidal friction (e.g. Malmberg et al. 2007; Fabrycky \& Tremaine 2007; Chatterjee et al. 2008; Nagasawa et al. 2008; Guillochon et al. 2010). Alternatively, it has been proposed that the orbit still reflects the orientation of the disk, with the stellar spin instead having moved away, either earlyon through magnetosphere-disk interactions (Lai et al. 2010) or later through elliptical tidal instability (Cébron et al. 2011). Distinguishing between these mechanisms needs additional obliquity measurements (e.g. Morton \& Johnson 2010).

Here we present spectroscopic observations of one transit of HAT-P-6b. This hot Jupiter transits a bright F star $(V=10.5)$ every 3.8 days and was discovered by Noyes et al. (2008, N08). Its mass is $1.06 \pm 0.12 M_{\text {Jup }}$ and its radius $1.33 \pm 0.06 R_{\text {Jup }}$.

\section{Radial velocity measurements with SOPHIE}

The 2010 August 21 transit of HAT-P-6b was observed with SOPHIE. This cross-dispersed, stabilized echelle spectrograph is dedicated to high-precision radial velocity measurements 
(Perruchot et al. 2008; Bouchy et al. 2009). It is fed by two optical fibers mounted at the focus of the 1.93-m telescope of the Haute-Provence Observatory (OHP, France). HAT-P-6 is bright enough for observation in the high-resolution mode of the spectrograph $(\lambda / \Delta \lambda=75000)$ and with a fast detector readout.

The observations were carried out three days before full Moon. This was anticipated not to affect the radial velocity accuracies adversely; however, thanks to the large radial velocity shift between HAT-P-6 $\left(-22.7 \mathrm{~km} \mathrm{~s}^{-1}\right.$, N08) and the Moon (close to the barycentric Earth radial velocity, which in the direction of HAT-P-6 was $16.3 \mathrm{~km} \mathrm{~s}^{-1}$ during the observations). We used the second fiber input to measure the sky background, and confirmed that its radial velocity was always shifted by at least $30 \mathrm{~km} \mathrm{~s}^{-1}$ from that of HAT-P-6. We therefore did not subtract the sky background, since this would have introduced additional noise.

We collected 41 measurements during the transit night $(\mathrm{JD} \simeq$ 2455430.5 ) between 20:10 and 03:40 UT. The sky was clear and the seeing stable around 1'.6. The airmass first decreased from $\sec z=1.8$ to 1.0 then increased to 1.1 at the end of the night. We adjusted the exposure times between 500 and $800 \mathrm{~s}$ to maintain a constant signal-to-noise ratio (SNR) of 39 per pixel at $550 \mathrm{~nm}$. Spectra of a thorium lamp were obtained at the beginning and end of the 7.5-h sequence, as well as three times during the sequence. These wavelength calibration exposures show that the spectrograph drifted by $\sim 2 \mathrm{~m} \mathrm{~s}^{-1}$ per hour. We linearly interpolated between the calibration points to correct for this drift.

We used the SOPHIE pipeline (Bouchy et al. 2009) to extract the spectra, to cross-correlate them with a G2-type numerical mask, and to measure the radial velocities through Gaussian fits to the cross-correlation functions (CCFs) (Baranne et al. 1996; Pepe et al. 2002). We discarded the four bluest orders of the SOPHIE spectra that have low SNR. This slightly improves the dispersion of the data around the model. Every spectrum produces a clean CCF with a peak contrast of $11.45 \pm 0.10 \%$ of the continuum, and a full width at half maximum $F W H M=$ $12.61 \pm 0.08 \mathrm{~km} \mathrm{~s}^{-1}$. The photon-noise uncertainty of the radial velocities, determined from the SNR of the spectra and the contrast and the FWHM of the CCF (see Boisse et al. 2010, for the details), is typically $\pm 18 \mathrm{~m} \mathrm{~s}^{-1}$. It is quite large by the SOPHIE standard because the star is an early-type and a moderately fast rotator $\left(V \sin i_{\mathrm{s}}=8.0 \pm 1.0 \mathrm{~km} \mathrm{~s}^{-1}\right.$ and $T_{\mathrm{eff}}=6570 \pm 80 \mathrm{~K}$; see below).

Figure 1 shows the SOPHIE velocities (see also the electronic table) as well as the orbital solution and HIRES data from N08. Outside transit, the SOPHIE data are compatible (within the expected arbitrary offset) with the HIRES orbit. During transit (lower panel), the SOPHIE data have obvious residuals relative to a Keplerian orbit, with a pattern opposite that expected for an aligned, prograde transit $\left(\lambda=0^{\circ}\right)$, red-shifted radial velocity in the first half of the transit followed by a symmetric blue shift. Here, the observed pattern of blue shifts during the first part of the transit and red shifts in the second part, instead, is characteristic of the RM anomaly with a retrograde orbit.

\section{Analysis}

We fited our SOPHIE data, together with the HIRES data from N08, to determine the sky-projected obliquity in the HAT-P-6 system. Interestingly, two HIRES measurements were serendipitously obtained in transits, on 2006 December 16 and 2007 July 4 (the two squares in the lower panel of Fig. 1). They agree with the SOPHIE measurements secured at the same orbital phases and thus with a retrograde orbit.

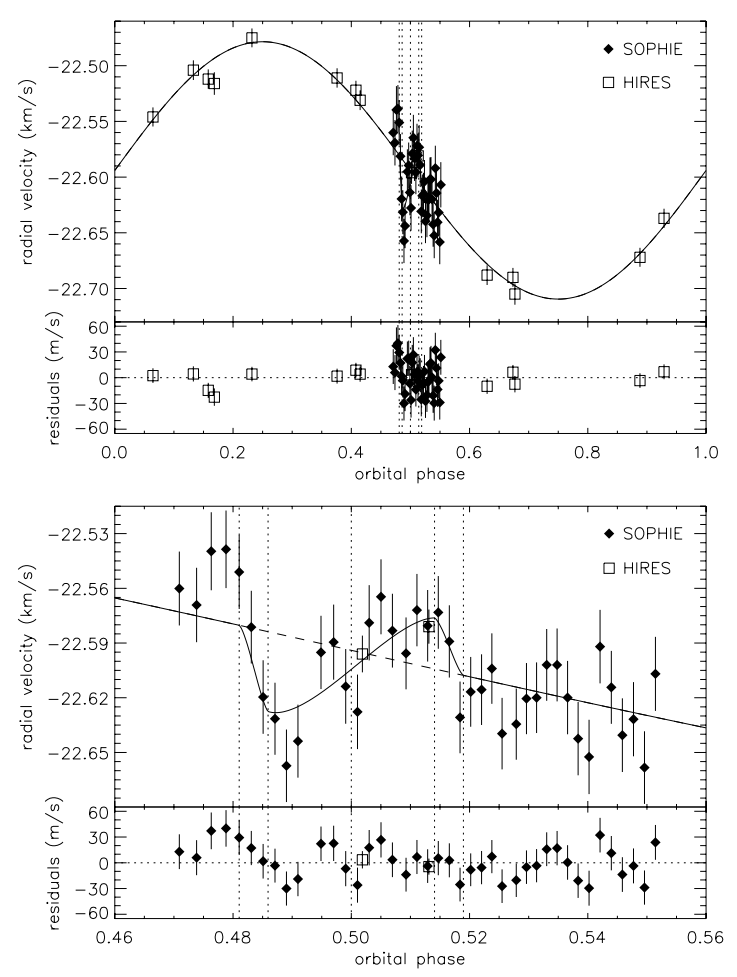

Fig. 1. Radial velocity measurements of HAT-P-6 as a function of orbital phase. Filled diamonds represent the SOPHIE data (this paper), and open squares the HIRES data (N08). The lower panel zooms on the transit phases. The dashed line represents the Keplerian fit (ignoring the transit phases), while the solid line represents the final fit including the model of the Rossiter-McLaughlin anomaly. In each panel, the residuals of the final fit are plotted below the data. The vertical dotted lines show the times of mid-transit, first, second, third, and fourth contacts. The fitted values are $V \sin i_{\mathrm{s}}=7.5 \pm 1.6 \mathrm{~km} \mathrm{~s}^{-1}$ and $\lambda=166^{\circ} \pm 10^{\circ}$.

The orbital elements of N08 determine the center of the 2010 August 21 transit, $T_{\mathrm{tr}}=2455430.4563 \pm 0.0018 \mathrm{BJD}$. This $\pm 2.6-$ min uncertainty is negligible for our analysis. The 22 SOPHIE measurements secured outside transit determine the systemic, barycentric stellar velocity as $\gamma_{\text {SOPHIE }}=-22.594 \pm 0.007 \mathrm{~km} \mathrm{~s}^{-1}$. The dispersion of their residuals from the orbital fit is $20.8 \mathrm{~m} \mathrm{~s}^{-1}$. The error bars in the electronic table and Fig. 1 include an extra $9-\mathrm{m} \mathrm{s}^{-1}$ uncertainty added in quadrature to the photon noise to obtain a reduced $\chi^{2}$ of 1 . Similarly the 13 HIRES measurements outside transit determine a systemic velocity $\gamma_{\text {HIRES }}=$ $-0.016 \pm 0.002 \mathrm{~km} \mathrm{~s}^{-1}$ (HIRES velocities are relative) and have a 9.6- $\mathrm{m} \mathrm{s}^{-1}$ dispersion around the Keplerian model. We quadratically added a 7.4- $\mathrm{m} \mathrm{s}^{-1}$ jitter to their error bars (instead of the $8.6 \mathrm{~m} \mathrm{~s}^{-1}$ adopted by N08) to obtain a $\chi^{2}$ of 1 . The Ca II activity level measured from the SOPHIE spectra, $\log R_{\mathrm{HK}}^{\prime}=$ $-5.03 \pm 0.10$, agrees reasonably with $\log R_{\mathrm{HK}}^{\prime}=-4.81$ measured by Wright (2005). It predicts a $10-\mathrm{m} \mathrm{s}^{-1}$ stellar jitter (Santos et al. 2000) consistent with these added dispersion terms.

We modeled the RM anomaly with the analytical firstmoment approach developed by Ohta et al. (2005). We adopted the above values of the systemic velocities and mid-transit time, and the following parameters for the circular orbit taken from N08: ratio of the planetary and stellar radii $R_{\mathrm{p}} / R_{*}=0.09338 \pm$ 0.00053 , scaled semi-major axis $a / R_{*}=7.69 \pm 0.22$, semiamplitude of the radial velocity variation $K=115.5 \pm 3.5 \mathrm{~m} \mathrm{~s}^{-1}$, orbit inclination $i_{\mathrm{O}}=85^{\circ} .51 \pm 0.35$, and orbital period $P=$ $3.852985 \pm 0.000005$ days. We computes a linear limb-darkening 


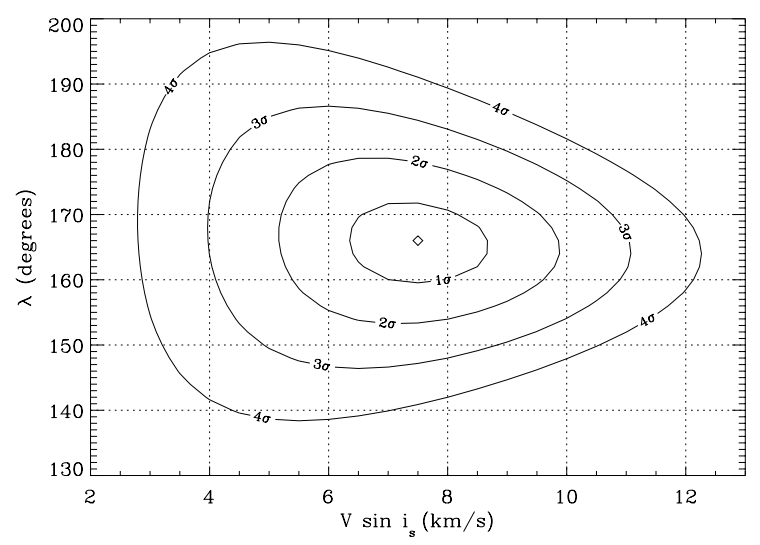

Fig. 2. $\chi^{2}$ formal isocontours for our modeling of the RossiterMcLaughlin effect as a function of $\lambda$ and $V \sin i_{\text {s }}$ (see Sect. 3), using the formula from Ohta et al. (2005) and the system parameters from N08. The diamond shows the best values.

coefficient $\epsilon=0.57 \pm 0.10$ in the $5300-6300 \AA$ wavelength range from a model atmosphere (Kurucz 1979).

The remaining two parameters of the Ohta et al. model are the sky-projected stellar rotational velocity $V \sin i_{\mathrm{s}}$ and the sky-projected spin-orbit angle $\lambda$. We determined these free parameters through a grid search, and derived formal confidence intervals from the $\chi^{2}$ isocontours plotted in Fig. 2 (Hébrard et al. 2002). The resulting values are $V \sin i_{\mathrm{s}}=7.5 \pm 1.2 \mathrm{~km} \mathrm{~s}^{-1}$ and $\lambda=166^{\circ} .0 \pm 6^{\circ} .1$. We separately propagated the effects of the uncertainties of the above fixed parameters on $V \sin i_{\mathrm{s}}$ and $\lambda$, and found a $\pm 1.1 \mathrm{~km} \mathrm{~s}^{-1}$ effect on $V \sin i_{\mathrm{s}}$, dominated by the uncertainties on $a / R_{*}, i$, and $\gamma_{\mathrm{SOPHIE}}$, and a \pm 8.5 effect on $\lambda$, mainly from the $\gamma_{\text {SOPHIE }}$ uncertainty. The other fixed parameters contribute negligibly to the $\lambda$ and $V \sin i_{\mathrm{s}}$ uncertainties. Quadratic addition of the two sources of uncertainties produces our final results: $V \sin i_{\mathrm{s}}=7.5 \pm 1.6 \mathrm{~km} \mathrm{~s}^{-1}$ and $\lambda=166^{\circ} \pm 10^{\circ}$.

The resulting $V \sin i_{\mathrm{s}}$ agrees with the $V \sin i_{\mathrm{s}}=8.0 \pm$ $1.0 \mathrm{~km} \mathrm{~s}^{-1}$, which we derive from the width of the CCF of HATP-6 (Boisse et al. 2010), as well as with $V \sin i_{\mathrm{s}}=8.2 \pm 1.0 \mathrm{~km} \mathrm{~s}^{-1}$ which N08 similarly derived from line broadening. The good agreement is somewhat surprising, since rotation broadens the lines of HAT-P-6 by significantly more than the SOPHIE resolution. As discussed by e.g. Hirano et al. (2010a) and Simpson et al. (2010), naive modeling of the RM anomaly could produce biased $V \sin i_{\mathrm{s}}$ measurements under such circumstances.

Figure 1 plots the full dataset and the final fitted model. The SOPHIE and HIRES velocities have dispersions around this model of $19.5 \mathrm{~m} \mathrm{~s}^{-1}$ and $9.0 \mathrm{~m} \mathrm{~s}^{-1}$, respectively. These are similar to the dispersions of the radial velocities measured outside transit around the Keplerian model, $20.8 \mathrm{~m} \mathrm{~s}^{-1}$ and $9.6 \mathrm{~m} \mathrm{~s}^{-1}$ (see above). Our RM model is thus a good description of the measurements with a reduced $\chi^{2}$ of unity.

\section{Discussion}

The orbit of HAT-P-6b is retrograde with respect to the spin of its host star (Fig. 3), but the true, unprojected angle $\psi$ between the two angular momenta remains uncertain. We have only measured the sky-projected spin-orbit angle, $\lambda=166^{\circ} \pm 10^{\circ}$, related to $\psi$ by $\cos \psi=\sin i_{\mathrm{s}} \cos \lambda \sin i_{\mathrm{o}}+\cos i_{\mathrm{s}} \cos i_{\mathrm{o}}$ (e.g. Fabrycky \& Winn 2009, whose notations and definitions we adopted: $i_{\mathrm{o}}$ is the inclination of the planetary orbit and $i_{\mathrm{s}}$ that of the stellar spin). The angle $i_{\mathrm{o}}$ is precisely known from the planetary transits but $i_{\mathrm{s}}$ remains unknown. There is no reasons to assume the star is seen

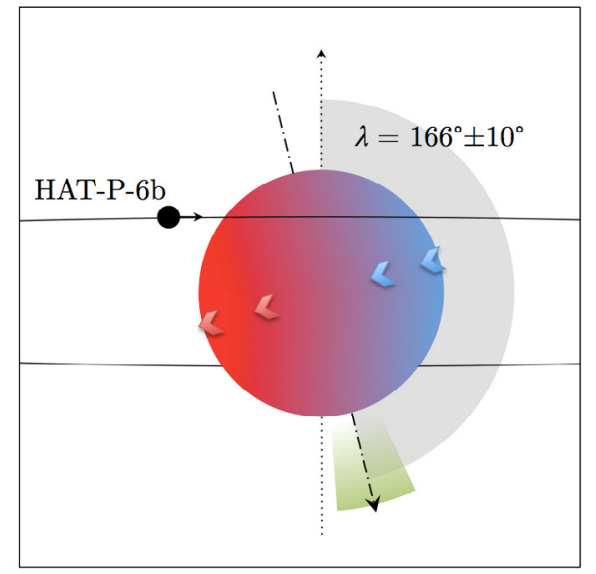

Fig. 3. Schematic view of the HAT-P-6 system from the Earth. The dotted and dashed-dotted lines represent the orbital angular momentum and the sky projection of the stellar spin axis. The red and blue hues on the star surface indicate the Doppler shift from stellar rotation. The skyprojected obliquity $\lambda$ and its uncertainty are displayed in gray and green.

edge-on $\left(i_{\mathrm{s}} \simeq i_{\mathrm{o}} \simeq 90^{\circ}\right)$, especially here where $\lambda \neq 0$ demonstrates some misaligment. Comparisons of the measured $V \sin i_{\mathrm{s}}$ to estimate the stellar rotation period $P_{\text {rot }}$ and radius $R_{*}$ can in principle constrain $i_{\mathrm{s}}$, but here with ambiguous results.

On one hand, the measured $R_{\mathrm{HK}}^{\prime}$ implies a short stellar rotation period, $P_{\text {rot }} \simeq 3.5$ days (Mamajek \& Hillenbrand 2008). From $R_{*}=1.46 \pm 0.06 R_{\odot}$ and $V \sin i_{\mathrm{s}}=8.0 \pm 1.0 \mathrm{~km} \mathrm{~s}^{-1}$, we obtain $P_{\text {rot }} / \sin i_{\mathrm{s}}=9.2 \pm 1.5$ days. Reconciling the two numbers requires an almost pole-on star, with $i_{\mathrm{s}} \simeq 20^{\circ}$ or $160^{\circ 1}$, implying $\psi \simeq 110^{\circ}$. Schlaufman (2010), on the other hand, predicts $V_{\mathrm{rot}}=9.1 \mathrm{~km} \mathrm{~s}^{-1}$ from the age and mass of HAT-P-6. This is similar to the measured $V \sin i_{\mathrm{s}}$, so would instead imply a more edgeon star, with $i_{\mathrm{s}} \simeq 60^{\circ}$ or $120^{\circ 2}$, and $\psi \simeq 145^{\circ}$. Neither approach is expected to be accurate. Here they give inconsistent estimates for $P_{\text {rot }}$ and therefore for $i_{\mathrm{s}}$ and $\psi$. Asteroseismology (Gizon \& Solanki 2003), polarization of magnetic dipoles (López Ariste et al. 2010), or accurate photometry may hopefully provide reliable $i_{\mathrm{s}}$ and $P_{\text {rot }}$ measurements. In the meantime, the value of $\psi$ remains unknown, except for its being above $90^{\circ}$.

HAT-P-6b is the seventh planet identified as having a retrograde orbit, after WASP-2b, 8b, 15b and 17b (Triaud et al. 2010; Queloz et al. 2010; Bayliss et al. 2010) and HAT-P-7b and 14b (Winn et al. 2009c, 2010a; Narita et al. 2009). Three planets also seem to have nearly polar orbits: CoRoT-1b (Pont et al. 2010), WASP-1b (Simpson et al. 2010), and HAT-P-11b (Winn et al. 2010b; Hirano 2010b). The last of them is also likely retrograde. In addition, HAT-P-11b is the only Neptune-mass planet with an obliquity measurement, with all other planetary systems with RM measurements being jovian mass planets.

The processes that produce tilted systems remain debated (see Sect. 1). Whether a single mechanism explains all close-in planets or whether different processes produce aligned and misaligned systems is unknown. Even whether the planetary orbits acquired obliquity or the stellar spin instead acquired tilt is uncertain. The mechanisms, in any case, cannot require conditions that are too narrowly specified, since tilted systems are common.

Schlaufman (2010) and Winn et al. (2010c) hypothesized that misaligned planets tend to orbit hot stars. The $6570 \pm 80 \mathrm{~K}$

\footnotetext{
1 The $i_{\mathrm{s}} \simeq 200^{\circ}$ and $340^{\circ}$ formal solutions can be excluded, since we know that the orbit is retrograde $\left(|\lambda|>\pi-i_{\mathrm{o}}\right)$.
}

${ }^{2}$ Here again, the retrograde orbit excludes $i_{\mathrm{s}} \simeq 240^{\circ}$ and $300^{\circ}$. 


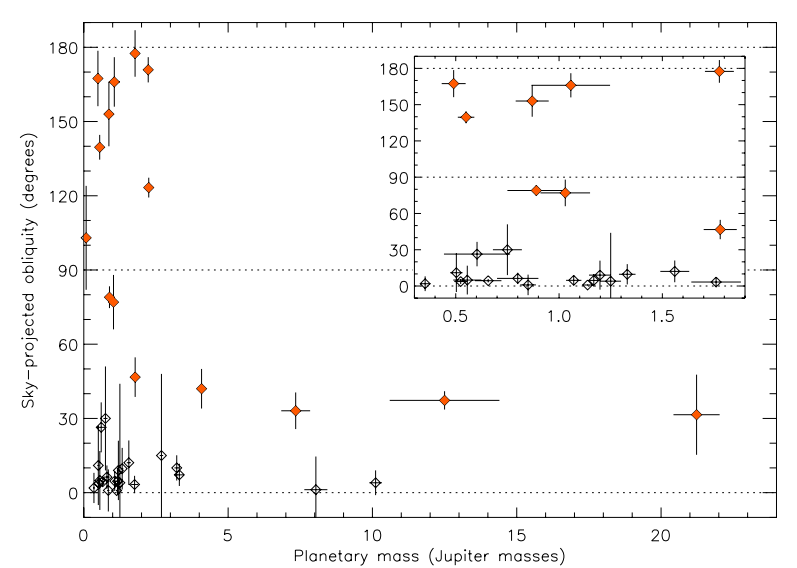

Fig. 4. Absolute value of $\lambda$, the sky-projected obliquity, as a function of planet mass $M_{\mathrm{p}}$ for the 37 planets with published RM measurements. Filled red markers denote systems with $|\lambda|>30^{\circ}$. The inset zooms on $0.3 M_{\text {Jup }}<M_{\mathrm{p}}<1.9 M_{\text {Jup }}$. The dotted lines mark aligned-prograde, polar, and aligned-retrograde systems $\left(|\lambda|=0^{\circ}, 90^{\circ}\right.$, and $180^{\circ}$, respectively). The 37 plotted planetary systems are the 21 ones compiled in Hébrard et al. (2010), plus XO-4 (Narita et al. 2010), WASP-1, 24, and 38 (Simpson et al. 2010), WASP-2, 4, 5, 15, and 18 (Triaud et al. 2010), WASP-8 (Queloz et al. 2010), WASP-19 (Hellier et al. 2011), HAT-P-4 and 14 (Winn et al. 2010a), HAT-P-8 (Simpson et al. 2010), HAT-P-11 (Winn et al. 2010b; Hirano 2010b), and HAT-P-6 (this work).

effective temperature of HAT-P-6 (N08) and the retrograde orbit of HAT-P-6b support this trend. As suggested by Johnson et al. (2009) and Hébrard et al. (2010), planetary mass apparently is also a key parameter. The seven retrograde and three polar planets all have masses below $3 M_{\text {Jup }}$. In that mass range, about a third of the planets with RM observations present such extreme obliquities, with the other two thirds having $\lambda \simeq 0^{\circ}$.

Measured planets with masses above $4 M_{\text {Jup }}$ are fewer, but they seem to have a different obliquity distribution: four (over six) of these massive planets are misaligned, but none with ultrahigh obliquities, i.e., none on a retrograde orbit and even none with $|\lambda|>50^{\circ}$. Just two of the massive planets show $\lambda \simeq 0^{\circ}$, HAT-P-2b (Winn et al. 2007; Loeillet et al. 2008) and WASP-18 (Triaud et al. 2010). Curiously, the period distribution of exoplanets detected from radial velocity surveys changes at a similar characteristic mass. There is a distinct lack of planets with $M_{\mathrm{p}}>4 M_{\text {Jup }}$ in short-period orbits ( $<100$ days), and radial velocity surveys detect no planets above that limit outside multiple star systems (Udry et al. 2003; Boisse et al. 2010).

The changes in the $\lambda$-distribution with planetary mass is illustrated in Fig. 4 by the 37 systems with published $\lambda$ and $M_{\mathrm{p}}$. The inset zooms on the Jupiter-mass planets, with their two thirds of aligned and one third strongly misaligned planets. The massive planets, in contrast, mostly show significant but moderate misalignments. This suggests that distinct mechanisms dominate for different planet masses, with a critical mass near 3.5 $M_{\text {Jup }}$. If a combination of planet scattering, Kozai mechanism, and tidal circularization explains most oblique, close-in planets (Nagasawa et al. 2008; Morton \& Johnson 2010), then planetary mass is a natural control parameter. These conclusions, however, currently suffer from small number statistics. Additional Rossiter-McLaughlin observations of massive planets are needed to confirm that most of them are tilted, and none of them are polar nor retrograde.
Acknowledgements. We thank the Haute-Provence Observatory staff who support the operation of SOPHIE. We acknowledge the support of the "Programme National de Planétologie" (PNP) of CNRS/INSU, the Swiss National Science Foundation, and the French National Research Agency (ANR-08-JCJC-010201 and ANR-NT05-4-44463). D.E. is supported by the CNES. A.E. is supported by a fellowship for advanced researchers from the Swiss National Science Foundation. I.B. and N.C.S. acknowledge the support by the European Research Council/European Community under the FP7 through Starting Grant agreement number 239953, as well as the support from Fundação para a Ciência e a Tecnologia (FCT) through program Ciência 2007 funded by FCT/MCTES (Portugal) and POPH/FSE (EC), and in the form of grants reference PTDC/CTEAST/66643/2006 and PTDC/CTE-AST/098528/2008.

\section{References}

Baranne, A., Queloz, D., Mayor, M., et al. 1994, A\&AS, 119, 373 Bayliss, D., Winn, J., Mardling, R., \& Sackett, P. 2010, ApJ, 722, L224 Boisse, I., Eggenberger, A., Santos, N. C., et al. 2010, A\&A, 523, A88 Bouchy, F., Hébrard, G., Udry, S., et al. 2009, A\&A, 505, 853 Cébron, D., Moutou, C., Le Bars, M., Le Gal, P., \& Fares, R. 2011 [arXiv: 1101.4531]

Chatterjee, S., Ford, E. B., Matsumura, S., \& Rasio, F. A. 2008, ApJ, 686, 580 Fabrycky, D., \& Tremaine, S. 2007, ApJ, 669, 1298 Fabrycky, D. C., \& Winn, J. N. 2009, ApJ, 696, 1230

Gizon, L., Solanki, S. K. 2003, ApJ, 589, 1009

Guillochon, J., Ramirez-Ruiz, E., \& Lin, D. N. 2010, ApJ, submitted [arXiv: 1012.2382]

Hébrard, G., Lemoine, M., Vidal-Madjar, A., et al. 2002, ApJ, 140, 103

Hébrard, G., Bouchy, F., Pont, F., et al. 2008, A\&A, 481, 52

Hébrard, G., Déssert, J.-M, Díaz, R. F., et al. 2010, A\&A, 516, A95

Hellier, C., Anderson, D. R., Collier Cameron, A., et al. 2011 [arXiv: 1101.3293]

Hirano, T., Suto, Y., Taruya, A., et al. 2010a, ApJ, 709, 458

Hirano, T., Narita, N., Shporer, A., et al. 2010b, PASJ, submitted [arXiv: 1009.5677$]$

Holt, J. R., 1893, A\&A, XII

Johnson, J. A., Winn, J. N., Albrecht, S., et al. 2009, PASP, 121, 1104

Kurucz, R. L. 1979, ApJS, 40, 1

Lai, D., Foucart, F., \& Lin, D. N. C. 2010, MNRAS, in press [arXiv: 1008.3148]

Lin, D. N. C., Bodenheimer, P., \& Richardson, D. C. 1996, Nature, 380, 606 Loeillet, B., Shporer, A., Bouchy, F., et al. 2008, A\&A, 481, 529

López Ariste, A., Asensio Ramos, A., Gonzlaez Fernanda, C., et al. 2010, A\&A, 527, A 120

Malmberg, D., Davies, M. B., \& Chambers, J. E. 2007, MNRAS, 377, L1

Mamajek, E. E., \& Hillenbrand, L. A. 2008, ApJ, 687, 1264

McLaughlin, D. B. 1924, ApJ, 60, 22

Morton, T. D., \& Johnson, J. A. 2010, ApJ, [arXiv: 1010.4025]

Moutou, C., Hébrard, G., Bouchy, F., et al. 2009, A\&A, 498, L5

Nagasawa, M., Ida, S., \& Bessho, T. 2008, ApJ, 678, 498

Narita, N., Sato, B., Hirano, T., \& Tamura, M. 2009, PASJ, 61, L35

Narita, N., Hirano, T., Sanchis, R., et al. 2010, PASJ, 62, L61

Noyes, R. W., Bakos, G. À, Torres, G., et al. 2008, ApJ, 673, L79 (N08)

Ohta, Y., Taruya, A., \& Suto, Y. 2005, ApJ, 622, 1118

Pepe, F., Mayor, M., Galland, F., et al. 2002, A\&A, 388, 632

Perruchot, S., Kohler, D., Bouchy, F., et al. 2008, SPIE Proc., 70140J

Pont, F., Hébrard, G., Irwin, J. M., et al. 2009, A\&A, 509, A695

Pont, F., Endl, M., Cochran, W. D., et al. 2010, MNRAS, 402, L1

Queloz, D., Eggenberger, A., Mayor, M., et al. 2000, A\&A, 359, L13

Queloz, D., Anderson, D., Collier Cameron, A., et al. 2010, A\&A, 517, L1

Rossiter, R. A. 1924, ApJ, 60, 15

Santos, N. C., Mayor, M., Naef, D., et al. 2000, A\&A, 361, 265

Schlaufman, K. C. 2010, ApJ, 719, 602

Simpson, E. K., Pollaco, D., Collier Cameron, A., et al. 2010, MNRAS, submitted [arXiv: 1011.5664]

Triaud, A., Collier Cameron, A., Queloz, D., et al. 2010, A\&A, 524, A25

Udry, S., Mayor, M., \& Santos, N. C. 2003, A\&A, 407, 369

Winn, J. N., Johnson, J. A., Peek, K. M. G., et al. 2007, ApJ, 665, L167

Winn, J. N., Johnson, J. A., Fabrycky, D., et al. 2009a, ApJ, 700, 302

Winn, J. N., Howard, A. W., Johnson, J. A., et al. 2009b, ApJ, 703, 2091

Winn, J. N., Johnson, J. A., Albrecht, S., et al. 2009c, ApJ, 703, L99

Winn, J. N., Howard, A., Johnson, J., et al. 2010a, AJ, 141, 63

Winn, J. N., Johnson, J. A., Howard, A. W., et al. 2010b, ApJ, 723, L223

Winn, J. N., Fabrycky, D., Albrecht, S., \& Johnson, J. A. 2010c, ApJ, 718, L145 Wright, J. T. 2005, PASP, 117, 657 